\title{
Guest editorial notes for selected papers from SOCA 2010
}

\author{
Jun Shen · Soo Dong Kim · Nalini Venkatasubramanian
}

Received: 24 April 2012 / Accepted: 26 April 2012 / Published online: 6 May 2012

(c) Springer-Verlag London Limited 2012

This special issue presents the extended and revised papers selected from the IEEE 2010 International Conference on Service-Oriented Computing and Applications (SOCA 2010), which was held in Perth, Australia. The SOCA conference is one of the major annual events sponsored by the IEEE Computer Society Technical Committee on E-Commerce (TCEC). SOCA 2010 received 111 high-quality paper submissions from 31 countries/regions. After a rigorous review process, the programme committee finally accepted 35 full papers for inclusion in the conference proceedings. After the conference, the Springer Journal of $<$ Service Oriented Computing and Applications $>$ invited the highly ranked papers to submit their extensions to this special issue. After two more rounds of rigorous reviews by prestigious researchers and thorough revisions by authors, only five papers have been accepted for publication. The authors of the accepted papers originated from 8 countries, and typically, 4 papers have been representative research outcome from international collaboration between academic institutions and/or industry partners.

Extensive research and development in the past few years has pushed SOC technology into numerous application areas including business processes, high-performance computing, Web-based services, embedded systems, and many others. With more and more services being moved into the "cloud", and the increasing prevalence of ubiquitous end devices that interface with the physical world, it is timely to

\footnotetext{
J. Shen $(\varangle)$

University of Wollongong, Wollongong, Australia

e-mail: jshen@uow.edu.au

S. D. Kim

Soongsil University, Seoul, Korea

N. Venkatasubramanian

University of California at Irvine, Irvine, CA, USA
}

re-examine the directions of SOC research and identify new research opportunities and challenges. Furthermore, ubiquitous devices, context-aware systems, and sensor networks are increasingly being used in areas such as transportation, health care, and disaster control, and this fact is making cyber-physical systems a challenging area for the SOC research community.

The selected papers cover a broad set of topics ranging from new application oriented work (e.g. real-time e-learning on the cloud) and theoretical breakthroughs (e.g. change impact analysis of business processes) to traditional issues such as service composition and aggregation. In the paper titled "Weighted Fuzzy Clustering for Capability-Driven Service Aggregation", Christoph Dorn and Schahram Dustdar proposed a new algorithm to aggregate services with different capabilities into clusters to meet users' QoS requirements. In "A Data Access Framework for Service-Oriented Rich Clients", Qi Zhao et al. presented a framework to tackle the overlooked complexity in managing the heterogeneous data storage mechanisms and cache strategies on traditional Web clients and servers. Although service composition has become a cliché problem, in the paper "Combining Horizontal and Vertical Composition of Services", Ralph Retter et al. innovatively introduced the namely "vertical service composition" and implemented it in an enterprise service bus (ESB) by combining concepts from dynamic service binding. Moreover, Yi Wang et al. revisited the change impact problem and proposed a new algorithm to analyse the newly defined change type and impact patterns in their paper titled "Change Impact Analysis in ServiceBased Business Processes". Finally, Tommaso Cucinotta and his team members reported their intriguing and novel work regarding "Virtualised e-Learning on the IRMOS Realtime Cloud", which is a critical part of IRMOS European Project. 
We believe the collection of papers offers an intriguing and diversified view on the latest progress in the SOC community and the applicability of the concepts to many application areas. We are indebted to the reviewers for their timely reviews. We also sincerely thank the journal's editorial team for their seasoning support. Their efforts have notably helped to present a high-quality special issue as it is. 